\title{
Insights into the composition of stroke thrombi: heterogeneity and distinct clot areas impact treatment
}

\author{
Rui-Gang Xu and Robert A. S. Ariëns
}

Discovery and Translational Science Department, Leeds Institute of Cardiovascular and Metabolic Medicine, University of Leeds, Leeds, UK

E-mail: ROBERT A. S. ARIËNS - r.a.s.ariens@leeds.ac.uk

doi:10.3324/haematol.2019.238816

T he structure of blood clots has recently become a hot topic in the scientific and medical literature. A search on PubMed for 'clot structure' results in $>1,000$ publications in the last 25 years, with $>400$ in the last five years alone. The reason for this surge in studies of the structure of blood clots is that dense clot structures that are resistant to fibrinolysis have been associated with both arterial and venous thrombosis (reviewed by Undas and Ariëns ${ }^{1}$ ), and lead to poor outcome in prospective studies of arterial $^{2}$ and venous ${ }^{3}$ thrombotic disease. Furthermore, mechanisms from cellular contributions from platelets, red blood cells (RBC) ${ }^{4}$ and white blood cells (neutrophils producing extracellular traps or $\mathrm{NET}^{5}$ ) to clot structure are increasingly being understood. However, many previous studies have focused on individual clot components and their roles in clot structure and function, largely using either in vitro or in vivo methodology. Holistic approaches to study clot structure in thrombi obtained from patients with thrombosis have until now been few and far between, and are increasingly needed to place studies of individual clot structures into clinical context.

In the current edition of Haematologica, Staessens et al. ${ }^{6}$ studied the internal organization of 177 thrombi collected from endovascularly treated ischemic stroke patients. The objective of this study was to gain further understanding of the composition of stroke thrombi by histologically analyzing the internal organization of their structural components, including fibrin, RBC, von Willebrand factor (vWF), platelets, leukocytes and DNA. Using bright field and fluorescence microscopy, the authors observed that stroke thrombi are very heterogeneous in nature in two ways. First, they differ distinctly from each other in size, shape, and color. Second, within each thrombus there is considerable heterogeneity, with different areas or segments of the thrombus demonstrating different structural components. Based on the major structural components that are dominant in the thrombi, they were classified into two distinct types: RBC- and platelet-rich areas of the thrombus (Figure 1). Platelet-rich areas are composed of dense fibrin structures, platelets, vWF, leukocytes and extracellular DNA, whereas composition of RBC-rich areas is less complex, with packed RBC and a thin fibrin meshwork filling the gaps in between the packed $\mathrm{RBC}$ as the main structural components. Staessens et al. ${ }^{6}$ further quantified the relative contribution of each type of thrombus area. It appears that the contribution of both types varies significantly across all thrombi analyzed. For most thrombi, both regions are dispersed throughout, although some thrombi have more clearly defined boundaries, with RBC-rich regions surrounded by platelet-rich regions. The findings obtained in this study provide interesting insight into the composition and internal organization of stroke thrombi and could be helpful in furthering our understanding of thrombolysis resistance and developing new therapies for acute ischemic stroke. Furthermore, knowledge of the structural composition of thrombi may be important for the relative success of mechanical thrombectomy.

This study makes important and valuable contributions to the previous efforts in the identification of thrombi composition, many of which were based on scanning electron microscopy ${ }^{7-12}$ There are a number of significant strengths of the current study. First, compared to previous studies, ${ }^{10,11}$ this is the first study to image thrombi obtained from patients with stroke with such detail, including visualization of multiple structural components of thrombi: fibrin, vWF, DNA and blood cells at the same time. Second, the use of immunofluorescence in this study overcomes limitations of conventional staining and generated stunning images, which allow for accurate localization of specific components and provide important structural insight of stroke thrombi both at cellular and molecular levels. In particular, compared with previous studies using conventional staining,,$^{7,12-14}$ more detailed organization and structural features of platelet, vWF and fibrin meshes in thrombi were demonstrated. Interestingly, the polyhedral morphology of RBC inside thrombi is also defined in these images, reminiscent of the tightly packed polyhedrocytes previously observed in thrombi formed in vivo and in vitro. ${ }^{15,16}$ Furthermore, the observation that extracellular DNA and leukocytes were located primarily in the platelet-rich areas, and close to the interface of these areas with others, emphasizes their potential crucial role in the rt-PA resistance observed in patients.

While this study makes big strides forward in our understanding of thrombus structure and function, some limitations remain. The first is that only microscopic methods were used to study the histological composition of thrombi, while there are no data on mechanical or functional properties of the thrombi. Previous studies on mechanical properties have shown that fibrin-rich clots have a higher friction than RBC-rich clots, ${ }^{17}$ and the increased percentage of $\mathrm{RBC}$ in the clot affects fibrin network heterogeneity and clot stiffness,$^{18}$ indicating that differences in thrombi composition may be strongly linked to the mechanical properties of these clots. The mechanical properties of thrombi may in turn impact on the degree of embolization on one hand, and the success rate of thrombus retrieval by endovascular thrombectomy on the other. A comprehensive study of the mechanical properties of thrombi in patients is needed to further our understanding of the possible correlations between 
thrombus composition and their mechanical properties, which would be advantageous in guiding the development of new generations of stent retrievers, as well as the selection of optimal thrombectomy therapy for patients. Another limitation of current studies regards the handling and storage of thrombus samples from patients. The thrombi obtained from thrombectomy are likely to undergo structural changes during or after the retrieval process. It is usually not possible to tell if the whole clot has been retrieved, or only fragments of it, and which parts represent the 'head' of the clot or the 'tail'. Furthermore, the internal organization and composition are probably modified by chemical fixation, air exposure or physical manipulation. A standard thrombus processing method needs to be developed to standardize those influences on thrombi during and after the retrieval process. ${ }^{19,20}$ In addition, the thrombi analyzed in this study only represent those that were successfully retrieved by thrombectomy, and were obtained from patients who received either prior rt-PA treatment or not. Future histological studies focusing on thrombi that are resistant to thrombectomy and rt-PA treatment would open a new window for advancing the treatment of acute ischemic stroke using intravascular approaches.
Intriguing new questions are also raised by the current study. For example, the role of polyhedrocytes observed in the thrombus remains poorly characterized. Polyhedrocytes are formed in contracted blood clots and thrombi due to the compression by activated contractile platelets pulling on fibrin. Remarkably, polyhedrocytes were first described in German by Gottlob et al. In 1970, ${ }^{21}$ Cynes et al. further characterized polyhedral erythrocytes, and described their occurrence in clots made in vitro and in thrombi obtained by thrombectomy from patients with myocardial infarction. ${ }^{15,16,22}$ The occurrence of polyhedrocytes in coronary thrombi was independently confirmed by Zalewski et al. ${ }^{23}$ These studies suggest that polyhedrocytes play important roles in thrombi, by forming a near impermeable seal that impairs diffusion of fibrinolytic enzymes into thrombi, leading to reduced thrombolysis. However, despite the multiple observations and efforts made by previous studies, detailed mechanisms of polyhedrocyte formation in contracted thrombi and their clinical and pathological implications are still underappreciated. Furthermore, thrombus contraction, usually associated with the presence of polyhedrocytes, is another interesting topic. It has been reported that patients with VTE and PE have significantly reduced

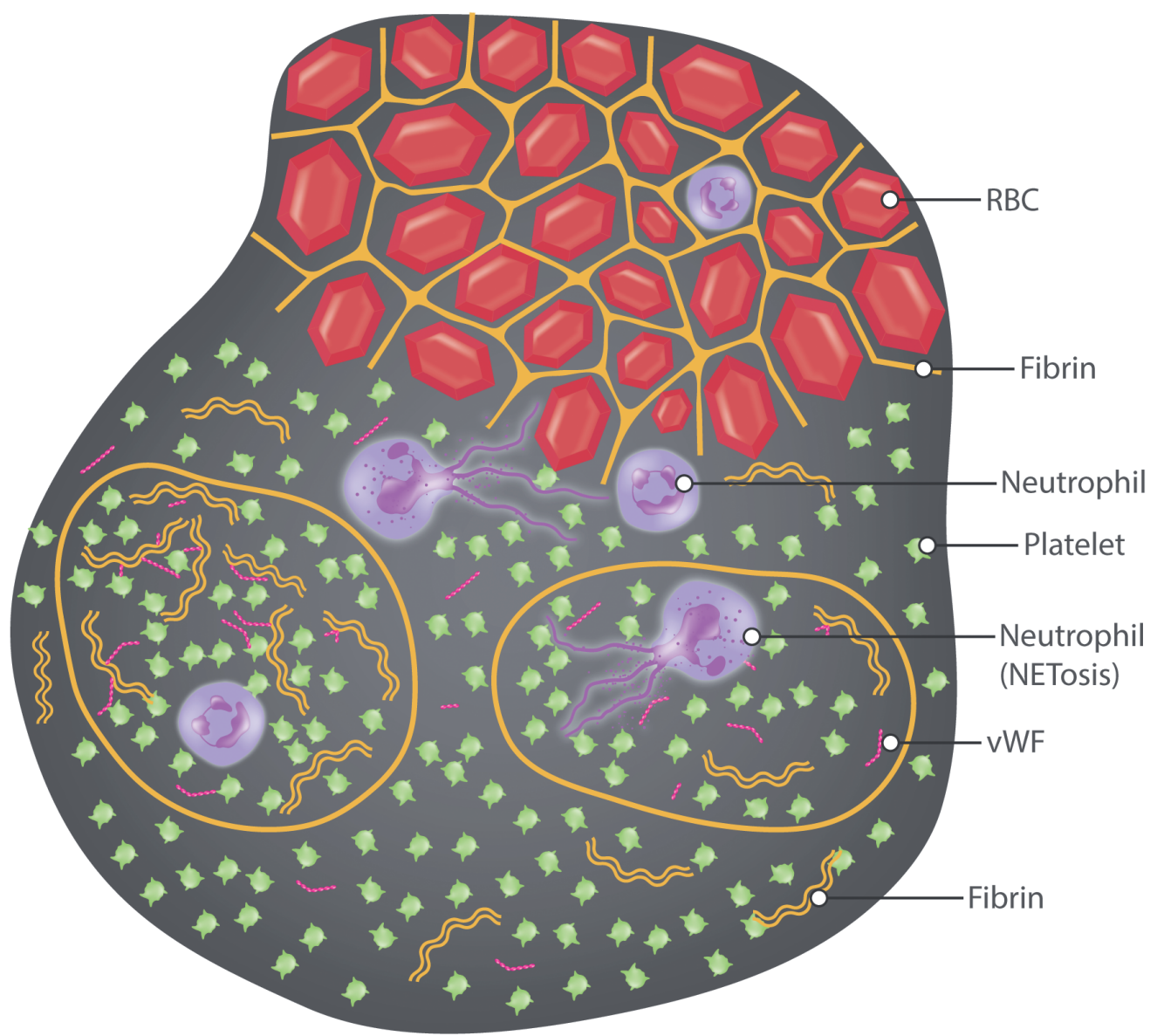

Figure 1. Schematic representation of the composition and organization of ischemic stroke thrombi as reported by Staessens et al. ${ }^{6}$ (Top) Red blood cell (RBC) rich area. This area mainly consists of packed RBC (polyhedrocytes) surrounded by thin fibrin fibers, which fill (or 'cement') the space between cells. Leukocytes (neutrophils) are also observed in these areas, but are less abundant. (Bottom) Platelet-rich area. Multiple structural components are found in this area, including platelets, thick fibrin bundles, von Willebrand factor (vWF), leukocytes (neutrophils) and extracellular DNA (due to NETosis). 
clot contraction compared to healthy donors, ${ }^{24}$ suggesting important associations between changes in the ability of blood clots to contract and the incidence of thromboembolism. These observations indicate polyhedrocytes as potential clinical markers of thromboembolism or targets for therapeutic intervention.

How the structure of the stroke thrombi in the report by Staessens et al. ${ }^{6}$ compares to other arterial or venous thrombi is another intriguing point. While arterial and venous thrombosis may be closely associated, ${ }^{25,26}$ they are triggered by different initiating mechanisms which may result in differences in the internal structure and composition of the thrombi. ${ }^{27}$ Arterial thrombi occur under high shear stress, leading to platelet-rich clots that form around ruptured atherosclerotic plaques and the damaged endothelium. In contrast, venous thrombi occur under low shear stress and mostly on an intact, although likely inflamed, endothelium. Compared to arterial thrombi, venous thrombi are thought to favor the formation of clots that are fibrin-rich, encapsulating a large number of $\mathrm{RBC}$ in addition to activated platelets. However, despite these differences in initiating mechanisms, recent studies on structural characteristics of arterial thrombi challenge the concept that arterial thrombi are platelet-rich and venous thrombi are RBC-fibrin rich, since the arterial thrombi also contained large amounts of fibrin and RBC in addition to platelets. ${ }^{16,23}$ The current study by Staessens et al. ${ }^{6}$ also shows significant areas that are RBC- and fibrin-rich in arterial stroke thrombi. Therefore, differences in the composition of arterial and venous thrombi are likely subtler and may not be as distinct with regards to relative fibrin, platelet and $\mathrm{RBC}$ contents as previously thought. Is the clot organization observed in this work for stroke thrombi also applicable to thrombi obtained from other types of thrombosis? More in-depth studies using thrombi obtained from other arterial or venous sources would help answer this very question.

The insightful imaging of thrombi from patients with stroke presented in this paper from our Belgian colleagues clearly contributes to our understanding of the cellular and molecular make-up of thrombi. It also sets an elegant example for future analysis of thrombi from other vascular beds. Once more data are generated regarding the structural heterogeneity of thrombosis in both the venous and arterial circulation, we will be able to associate these findings with in vitro blood clot structure from systemic samples, providing tantalizing opportunities for new diagnostic tools. In addition, once the functional consequences of different thrombi structures on the behavior of these thrombi, their stability and future outcome is better documented, we should be able to improve interventional and medical treatment of thrombosis, and explore theranostics or other improved personalized approaches based on the nature of the thrombus that needs to be removed.

\section{References}

1. Undas A, Ariëns RA. Fibrin clot structure and function: a role in the pathophysiology of arterial and venous thromboembolic diseases. Arterioscler Thromb Vasc Biol. 2011;31(12):e88-e99.

2. Sumaya W, Wallentin L, James SK, et al. Fibrin clot properties inde- pendently predict adverse clinical outcome following acute coronary syndrome: a PLATO substudy. Eur Heart J. 2018;39(13):1078-1085.

3. Siudut J, Swiat M, Undas A. Altered fibrin clot properties in patients with cerebral venous sinus thrombosis: association with the risk of recurrence. Stroke. 2015;46(9):2665-2668.

4. Weisel J, Litvinov R. Red blood cells: the forgotten player in hemostasis and thrombosis. J Thromb Haemost. 2019;17(2):271-282.

5. Martinod K, Wagner DD. Thrombosis: tangled up in NETs. Blood. 2014;123(18):2768-2776.

6. Staessens S, Denorme F, François $O$, et al. Structural analysis of ischemic stroke thrombi: histological indications for therapy resistance. Haematologica. 2019;105(2):498-507.

7. Silvain J, Collet JP, Nagaswami C, et al. Composition of coronary thrombus in acute myocardial infarction. J Am Coll Cardiol. 2011;57(12):1359-1367.

8. Sadowski M, Ząbczyk M, Undas A. Coronary thrombus composition: links with inflammation, platelet and endothelial markers. Atherosclerosis. 2014;237(2):555-561.

9. Ramaiola I, Padró T, Peña E, et al. Changes in thrombus composition and profilin-1 release in acute myocardial infarction. Eur Heart $J$. 2014:36(16):965-975

10. Marder VJ, Chute DJ, Starkman S, et al. Analysis of thrombi retrieved from cerebral arteries of patients with acute ischemic stroke. Stroke. 2006;37(8):2086-2093.

11. Simons N, Mitchell P, Dowling R, Gonzales M, Yan B. Thrombus composition in acute ischemic stroke: a histopathological study of thrombus extracted by endovascular retrieval. J Neuroradiol. 2015;42(2):86-92.

12. Fitzgerald S, Mereuta OM, Doyle KM, et al. Correlation of imaging and histopathology of thrombi in acute ischemic stroke with etiology and outcome. J Neurosurg Sci. 2019;63(3):292-300.

13. Hoshiba Y, Hatakeyama K, Tanabe T, Asada Y, Goto S. Co-localization of von Willebrand factor with platelet thrombi, tissue factor and platelets with fibrin, and consistent presence of inflammatory cells in coronary thrombi obtained by an aspiration device from patients with acute myocardial infarction. J Thromb Haemost. 2006;4(1):114120.

14. Shin JW, Jeong HS, Kwon HJ, Song KS, Kim J. High red blood cell composition in clots is associated with successful recanalization during intra-arterial thrombectomy. PloS One. 2018;13(5):e0197492.

15. Tutwiler V, Mukhitov AR, Peshkova AD, et al. Shape changes of erythrocytes during blood clot contraction and the structure of polyhedrocytes. Sci Rep. 2018;8(1):17907.

16. Cines DB, Lebedeva T, Nagaswami C, et al. Clot contraction: compression of erythrocytes into tightly packed polyhedra and redistribution of platelets and fibrin. Blood. 2014;123(10):1596-1603

17. Gunning GM, McArdle K, Mirza M, Duffy S, Gilvarry M, Brouwer PA. Clot friction variation with fibrin content; implications for resistance to thrombectomy. J Neurointerv Surg. 2018;10(1):34-38.

18. Gersh KC, Nagaswami C, Weisel JW. Fibrin network structure and clot mechanical properties are altered by incorporation of erythrocytes. Thromb Haemost. 2009;102(6):1169-1175.

19. De Meyer SF, Andersson T, Baxter B, et al. Analyses of thrombi in acute ischemic stroke: a consensus statement on current knowledge and future directions. Int J Stroke. 2017;12(6):606-614

20. Staessens S, Fitzgerald S, Andersson T, et al. Histological stroke clot analysis after thrombectomy: Technical aspects and recommendations. Int J Stroke. 2019:1747493019884527.

21. Gottlob R, Pötting U, Schattenmann G. [Morphologische Untersuchungen zur Frage der Retraktion, der sekundären Quellung und der Lysierbarkeit von Thromben]. Langenbeck's Archives of Surgery. 1970;327:1209-1210.

22. Ariëns RA. A new red cell shape helps the clot. Blood. 2014;123 (10):1442-1443.

23. Zalewski J, Bogaert J, Sadowski M, et al. Plasma fibrin clot phenotype independently affects intracoronary thrombus ultrastructure in patients with acute myocardial infarction. Thromb Haemost. 2015;113(6):1258-1269.

24. Peshkova AD, Malyasyov DV, Bredikhin RA, et al. Reduced contraction of blood clots in venous thromboembolism is a potential thrombogenic and embologenic mechanism. TH Open. 2018;2(1):e104e115.

25. Franchini M, Mannucci PM. Association between venous and arterial thrombosis: clinical implications. Eur J Intern Med. 2012;23(4): 333-337.

26. Franchini M, Mannucci PM. Venous and arterial thrombosis: different sides of the same coin? Eur J Inter Med. 2008;19(7):476-481.

27. Koupenova M, Kehrel BE, Corkrey HA, Freedman JE. Thrombosis and platelets: an update. Eur Heart J. 2016;38(11):785-791. 